\title{
VIRTUAL REALITY IN MEDICINE: A BRIEF OVERVIEW AND FUTURE RESEARCH DIRECTIONS
}

rewiev paper

(1) University School of Physical Education in Wroclaw

DOI: https://doi.org/10.5114/hm.2019.83529

\section{JUSTYNA MAZUREK ${ }^{1}$, PAWEŁ KIPER ${ }^{2}$, BŁAŻEJ CIEŚLIK ${ }^{3}$, SEBASTIAN RUTKOWSKI ${ }^{4}$, KRZYSZTOF MEHLICH ${ }^{5,6}$, ANDREA TUROLLA ${ }^{2}$, JOANNA SZCZEPAŃSKA-GIERACHA ${ }^{7}$}

${ }^{1}$ Department and Division of Medical Rehabilitation, Wroclaw Medical University, Wroclaw, Poland

${ }^{2}$ Laboratory of Neurorehabilitation Technologies, Fondazione Ospedale San Camillo IRCCS, Venice, Italy

${ }^{3}$ Institute of Physical Education, Tourism and Physiotherapy, Faculty of Pedagogy, Jan Dlugosz University, Czestochowa, Poland

${ }^{4}$ Institute of Physiotherapy, Faculty of Physical Education and Physiotherapy, Opole University of Technology, Opole, Poland

${ }^{5}$ Upper Silesian Rehabilitation Centre 'Repty,' Tarnowskie Gory, Poland

${ }^{6}$ Faculty of Physiotherapy, The Jerzy Kukuczka Academy of Physical Education, Katowice, Poland

${ }^{7}$ Department of Physiotherapy, University School of Physical Education, Wroclaw, Poland

\section{ABSTRACT}

Virtual reality (VR), also called phantomatics, is an image of artificial reality created entirely in three-dimensional graphics with the use of information technology. VR makes it possible to experience the imaginary world as if it was real, where sensory data are delivered to the brain through a specialized system. The purpose of virtual medicine is to minimize direct contact and impact on human body during treatment. Taking into consideration the increasing accessibility of high quality electronic devices, their immense computing powers, and the continuously developing Internet infrastructure, the advancement in this area is only a matter of time. Therefore, the aim of the study was to identify the applications of VR in medicine, focusing on the areas of psychiatry and rehabilitation and considering guidelines for future research.

Key words: virtual reality, VR goggles, psychiatry, rehabilitation

'What does virtual reality mean?

Virtual reality is practically real but it isn't' Robert Altman

'Virtual reality is dreams' Morton Heilig

\section{Introduction}

Recent years have seen a significant progress in medical technology, among others with regard to new, minimally invasive methods closely linked to virtual reality (VR) (the so-called virtual medicine). VR is based on creating a computer representation of objects, spaces, and events. It is, therefore, a simulation of real situations, a high-quality user interface with real-time sim- ulation and interaction through multiple sensory channels. At present, there are many different ways to create a fully or partially virtual world [1]. Depending on what real and virtual objects are presented in the image, there are four basic categories: (1) reality, the real world; (2) augmented reality, where computer-generated data merge into a real-world image; (3) augmented virtuality, where real-life data are merged into a computer-generated world; and (4) VR, where the world is created entirely by a computer [1, 2]. It must be noted that the creation of a fully virtual world requires not only images, but also the stimulation of other senses: sound, smell, taste, and touch. The purpose of virtual medicine is to minimize direct contact and impact on human body. It may therefore

Correspondence address: Justyna Mazurek, Department and Division of Medical Rehabilitation, Wroclaw Medical University, ul. Borowska 213, 50-556 Wrocław, Poland, e-mail: justyna.mazurek@umed.wroc.pl

Received: October 5, 2018

Accepted for publication: February 2, 2019

Citation: Mazurek J, Kiper P, Cieślik B, Rutkowski S, Mehlich K, Turolla A, Szczepańska-Gieracha A. Virtual reality in medicine: a brief overview and future research directions. Hum Mov. 2019;20(3):16-22; doi: https://doi.org/10.5114/ hm.2019.83529. 
be used by students of medicine who want to learn new techniques, as well as by experienced doctors and therapists who wish to provide their patients with optimal, minimally invasive, but effective and safe treatment methods. VR offers new ways to develop social skills, socialize and interact with other people via customizable, realistic, 3D, fully textured and animated avatars.

\section{Purpose of the study}

This study attempts to show the applications of VR in medicine, focusing on the areas of psychiatry and rehabilitation and future research directions.

\section{History of virtual reality}

The term 'virtual reality' was coined by Jaron Lanier, a writer, musician, visual artist, and computer scientist, who first used it in 1986 in a discussion with Scott Fisher regarding Fisher's work on the so-called virtual environment. An earlier term, 'artificial reality, was suggested by an American computer scientist and artist Myron Krueger, who was hence called the pioneer of VR (1969) [2]. Krueger is also the creator of a VR system developed in 1992 under the name 'cave automatic virtual environment' (CAVE), where a stereoscopic image was projected onto the floor and walls of a cubic room. This technology was adopted for commercial use as early as in the 1970s. The first application of VR in healthcare dates back to the beginning of the 1990s. It stemmed from the need to visualize complex medical data, especially when planning surgical treatment [3]. In 2009, the International Society for Virtual Rehabilitation was established, whose task was to create a common ground for cooperation between engineers, scientists, and clinicians interested in the introduction of new technologies in motor, psychological, cognitive, and social rehabilitation. Currently, VR is extensively used in medicine, from teaching and training (anatomical and functional imaging, laparoscopic simulators), through surgery (planning and supporting surgical procedures, virtual endoscopy), psychology, rehabilitation, to such fields as telemedicine [4].

\section{Virtual reality in scientific research}

A number of well-developed VR techniques from the macroscopic world have been adopted to the world of molecules and nanomolecules owing to similar techniques of three-dimensional visualization of objects [5].
As for interaction with molecules, virtual gloves allow to literally 'catch' individual atoms (the so-called virtual touch), stretch the bonds between them, and physically feel their strength. Therefore, it has now become possible to work interactively with computergenerated, virtual three-dimensional objects which are subject to the encoded laws of physics. This allows scientists to understand the molecular biology of various diseases, including Alzheimer's, Parkinson's, or Huntington's disease. In addition, integrated three-dimensional systems have been developed for drug design, allowing to create and optimize a protein model (e.g. the Molecular Operating Environment by the Chemical Computing Group) [6]. In order to increase the computing capacity, Internet projects have been established to study, among others, the folding processes and the physicochemical properties of proteins, where hundreds of thousands of personal computers throughout the world connect over the Internet with servers (e.g. at the Stanford University: Folding@Home, or the Washington University: Rosetta@Home), collect the data for calculations, and return the obtained results. Such an organization of work allows for an unlimited participation of volunteers and continuous development of the project $[7,8]$.

\section{Virtual reality in psychiatry}

The idea of using VR technology to treat various mental disorders was first introduced at the Clark Atlanta University in 1992. Since then, its effectiveness, especially in the therapy of phobias [9], has been confirmed in numerous experiments. Literature knows abundant accounts of VR being used to treat phobias, which are among the most common mental disorders $[10,11]$. In traditional cognitive behavioural therapies, the key is a progressive exposure to stimuli that trigger symptoms of the phobia, which involves the patients imagining themselves in certain difficult situations. However, this type of exposure is not always effective, mainly because many patients find it difficult to imagine an aversive situation. Therapy making use of the possibilities created by VR proves to be effective in this type of disorder, as it allows the patients to be exposed to a variety of stimuli in a safe environment.

Recently, reports have appeared suggesting that VR may also be effective in the treatment of depressive disorders. In 2014, a meta-analysis was carried out regarding publications that used VR-based computer games to improve mood. Overall, 19 publications were analysed involving 1474 participants of various age. 
The depression therapy used computer games which induced a feeling of relaxation in the participants, encouraged physical activity, or applied the psychoeducation method. The study showed that this type of intervention significantly lowered the severity of depressive symptoms in the studied group [12]. In another study, Valmaggia et al. [13] analysed 24 publications involving 1305 participants with mental disorders such as depression, eating disorders, or post-traumatic stress disorder. They concluded that by using immersive VR, one can achieve similar therapy results as with conventional treatments for these types of disorders. VR can produce specific situations that can be therapeutically helpful if implemented in the right way but remain near impossible to recreate in real life. VR allows repeated and immediately available treatment input. With VR, patients with mental disorders can enter simulations of difficult situations and be coached in the appropriate responses, based upon the best theoretical understanding of the specific psychiatric illness. Because the simulations can be graded in difficulty and experienced for many times until the right learning is achieved, patients will face difficult situations with much more ease in VR than in real life. Additionally, VR offers people with psychiatric diagnoses an opportunity to try out new therapeutic strategies [9, 13].

\section{Virtual reality in rehabilitation}

The last decade has seen a rapid development of VR and interactive video games used especially to induce motor function recovery of paretic upper limbs in stroke patients. We can currently talk about the so-called 'virtual rehabilitation,' which is an innovative method of improving motor function in people with different dysfunctions with the use of modern computer technologies based on VR [14]. Virtual rehabilitation involves both activities carried out 'here and now' and therapy conducted at a distance, called telerehabilitation. A number of reports have been published on this subject, including meta-analyses [14-22] evaluating the efficacy of different technical solutions. Standen et al. [20] found that VR-based games might also be used at home to help improve motor function in upper limbs after a stroke. Thanks to a variety of tasks, images, and effects that can make conventional motor treatment more engaging, rehabilitation with VR guarantees a greater involvement of the patient and makes them more motivated to exercise regularly. To date, several studies have described the use of VR systems to improve upper limb function within 6 months from stroke onset, but there is a continuous need for further research in this area.

A valuable publication is a systematic review of the Cochrane database regarding studies that used VR in stroke patients, presented in 2015 by Laver et al. [23]. The primary aim of the review was to determine the efficacy of VR in improving the motor function of the upper limb. It also assessed the impact of VR on improving locomotion, cognitive functions, and self-reliance in performing basic activities of the daily life. Eventually, having analysed 8244 publications, the review included 37 studies that met the requirements of randomized or quasi-randomized studies carried out between 2004 and 2014, with a total of 1019 participants. VR was found significantly superior to conventional rehabilitation treatments used to improve functions of the upper limb after stroke (standardized mean difference [SMD]: 0.28, 95\% confidence interval [CI]: 0.08-0.49). Moreover, VR application significantly improved the patients' independence in daily activities compared with traditional treatment methods (SMD: 0.43, 95\% CI: 0.18-0.69). The authors of the studies reported rare adverse effects, which included dizziness and headaches.

In 2016, Saposnik et al. [24] conducted a randomized, multicentre, single-blind trial within the EVREST project, which compared 2 groups of patients up to 3 months after stroke $(n=101)$, using non-immersive VR - the Nintendo Wii game system $(n=54)$ and the so-called recreational activity $(n=47)$. Patients in both groups were subjected to 60-minute training sessions over a period of 2 weeks (10 sessions). Both after 2 and after 4 weeks from the beginning of exercises, control measurements using the Wolf Motor Function Test and the Chedoke Arm and Hand Activity Inventory showed that the subjects in the study group (VR) obtained similar final results as the control group, which means that VR is a method equivalent to traditional improvement treatments. This is an important conclusion since in the coming years, the price of computer rehabilitation systems will decrease, while human work will be increasingly expensive owing to shortages of qualified medical staff. VR will be particularly useful in the ageing society, where the demand for medical services will quickly exceed the supply.

The development of $\mathrm{VR}$ in recent years has resulted in the emergence of many different systems, including the CAVE-type environments. The CAVE system, also called a 'virtual cave,' allows for placing the patient in the 'centre of the virtual world' [25]. The user has the impression of being present in the world generated 
by computers. A multi-wall projection of stereoscopic images together with 3D glasses allow the patient to see objects that do not exist in the real world. Owing to an active motion tracking system, the user can navigate inside the cave while controlling the projection of the images displayed in the computer-created world. The use of VR also proves beneficial in preventing falls among the elderly. Da Silva et al. [26] proved that active video game play promoted a similar reduction in blood pressure compared with traditional walking exercise, with the advantage of inducing reduction in the sympathetic inflow to the heart. Rehabilitation using VR appeals to elderly patients because it gives them the possibility to diversify treatment by introducing alternative forms of activity. However, there is still a demand for more randomized clinical studies that would confirm the efficacy of this form of activity, especially in patients suffering from the frailty syndrome, who are the largest group of beneficiaries of this type of rehabilitation $[27,28]$.

\section{Future research directions}

Whatever limits the real world imposes on us, the virtual world is its ideal, unlimited reflection, and creates a space where the impossible becomes possible. A space where modern technological solutions generate new reality. VR is no longer an abstract concept, accessible only to advanced enthusiasts of computer games. We can currently see VR being used in many areas of daily life, including medicine. In the near future, VR will most likely become the most common method of teaching and training young surgeons, as well as helping experienced practitioners to acquire new skills. It is predicted that the next step will be combining VR with holographic projection, thus improving the three-dimensional image [29]. The combination of VR with autonomous cybernetic systems, whose possibilities include the ability to selfregulate and self-sustain their existence, will result in the emergence of autonomous surgical robots, autonomous rehabilitation systems for disabled people, autonomous education systems, and complex virtual research software [3]. VR is a very active field of research. However, the application of VR techniques in medicine and rehabilitation requires overcoming many barriers, not only financial, but also mental.

The aging of the societies of the developed and developing countries is a fact and it is going to deepen in the upcoming decade. There is a lack of both medical staff and caregiver forces; therefore, more and more hope is being reposed in modern technologies every year.
At the same time, there is an extensive discussion (in medical, social, and ethical fields) on the extent to which robots and VR can replace man in the treatment, rehabilitation, and taking care of ill, disabled, and old people $[22,30]$. In the opinion of the authors of this paper, the answer is obvious: the participation of new technologies will grow year by year. Taking into account the increasing accessibility of high quality electronic devices, their immense computing powers, and the continuously developing Internet infrastructure, the advancement in this area is only a matter of time. That is why it is worth considering guidelines of future research. Decreasing price, leading to greater accessibility of VR goggles offering total immersion in the virtual world, opens new possibilities.

VR glasses enable the display of high-resolution images with great picture quality $(90 \mathrm{~Hz})$. The applied organic light-emitting diode (OLED) display inside the glasses allows for highly accurate colour replication. Numerous sensors placed in the glasses (such as a gyroscope, accelerometer, magnetometer) are supported by dedicated software that processes the sensor data and instantly transfers movements of the user's head into the virtual scenario. With manipulators, hand movements of the person being rehabilitated may be precisely transferred into the VR world. The sensors positioned on the surface of controllers are detected in space, similarly to the glasses. The hand movement mapping is precise enough to enable automatic computer analyses, monitoring, for instance, the progress of the patient's rehabilitation. The projection of the image displayed inside the VR helmet is possible thanks to advanced methods of rendering (generating) threedimensional graphics. At present, the quality of the computer-generated image, in real time, is very close to photorealistic quality. The entire experience is made whole by the surrounding sound effects. Such a system enables its user to achieve a total immersion in the virtual world. A team of software developers, graphic designers, and musicians are capable to create practically any virtual environment so that it is interactive, operated with the above-mentioned controllers intuitively and with unprecedented precision, also by persons with reduced mobility. Thanks to VR it is possible to transfer a patient in the course of rehabilitation, who often moves in a wheelchair, into any environment and conduct therapy without barriers or risk factors, which at this stage of rehabilitation would be impossible to overcome in the patient's real world (Figure 1).

However, a question arises whether the elderly will be willing to use such modern equipment as VR goggles. A pilot study [own unpublished results] financed by 


\section{HUMAN MOVEMENT}

J. Mazurek et al., Virtual reality in healthcare

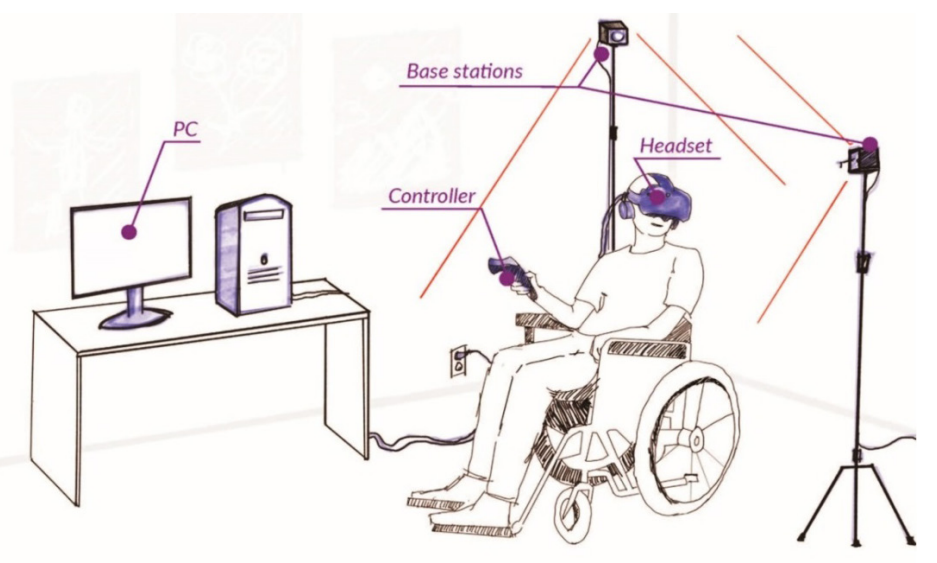

Figure 1. An example of a virtual reality system with goggles

the Polish National Centre of Research and Development, Action 1.2 Sector 'R\&D programmes' within the European Smart Growth Operational Programme for years 2014-2020, carried out between June 2017 and June 2018 in Polish nursing homes and rehabilitation facilities, showed that these concerns were unfounded. The study involved 52 participants over the age of 60 (mean age: $71.33 \pm 5.5$ years), who assessed the level of satisfaction associated with using VR goggles, with particular focus on the reality of the created world, as well as their impressions connected with the virtual world experience and the willingness to participate in regular VR goggle sessions. All participants reported a very high level of satisfaction associated with using the VR goggle technology. The patients referred to the reality of the computer-created world with great enthusiasm and were very keen to perform all instructions and tasks in the virtual environment. All participants declared the willingness to use this type of add-on treatment in the future to supplement their daily therapy. The study has shown that the mental barriers concerning a possible reluctance to the use of VR by elderly people exist primarily in the minds of younger people. What is more, it has been observed that the more monotonous, repetitive, predictable, and devoid of stimuli the real life of a senior is (as in the case of long-term nursing homes), the more interest or even fascination such a person shows with regard to VR environment.

\section{Conclusions}

In view of the demographic and cultural changes that are currently taking place in many countries and the resulting need for new long-term care centres for the elderly, the use of VR environment in these types of facilities seems justified and necessary. Taking into consideration the prevalence of mood disorders coexisting with disabilities in old patients rehabilitated in round-the-clock facilities or living in nursing homes, it appears very important to combine therapeutic and rehabilitation aspects to increase treatment efficacy. The virtual world can liven up the daily monotony and, depending on the needs of the patient, either foster calmness and psychophysical relaxation, or, on the contrary, activate and energize its user. Now, all is in the hands of research teams made up of specialists in various fields (psychologists and psychotherapists, physiotherapists, occupational therapists, doctors, and of course software developers, computer scientists, and IT specialists). Their commitment, knowledge, and creativeness will determine the development of the VR sector dedicated to elderly people with various health conditions and various needs. Our team's pilot study shows that this area definitely provides ample scope for development.

\section{Ethical approval}

The conducted research is not related to either human or animal use.

\section{Disclosure statement}

No author has any financial interest or received any financial benefit from this research.

\section{Conflict of interest}

The authors state no conflict of interest.

\section{Funding}

Polish National Centre of Research and Development, Action 1.2 Sector 'R\&D programmes' within the European Smart Growth Operational Programme for years 2014-2020, grant No. POIR.01-02.00-00$0134 / 16$.

\section{References}

1. Cipresso P, Giglioli IAC, Raya MA, Riva G. The past, present, and future of virtual and augmented reality research: a network and cluster analysis of the literature. Front Psychol. 2018;9:2086; doi: 10.3389/fpsyg.2018. 02086.

2. Ma JY, Choi JS. The virtuality and reality of augmented reality. J Multimed. 2007;2(1):32-37; doi: 10.4304/ jmm.2.1.32-37.

3. Graur F. Virtual reality in medicine - going beyond the limits. In: Lányi CS (ed.), The thousand faces of virtual reality. London: IntechOpen; 2014; 23-35.

4. Matthews D. Virtual-reality applications give science a new dimension. Nature. 2018;557(7703):127-128; doi: 10.1038/d41586-018-04997-2. 
5. Krieger E, Joo K, Lee J, Lee J, Raman S, Thompson J, et al. Improving physical realism, stereochemistry, and side-chain in homology modeling: four approaches that perform well in CASP8. Proteins. 2009;77(Suppl 9): 114-122; doi: 10.1002/prot.22570.

6. Liwo A, Ołdziej S, Czaplewski C, Kleinerman DS, Blood P, Scheraga HA. Implementation of molecular dynamics and its extensions with the coarse-grained UNRES force field on massively parallel systems: toward millisecond-scale simulations of protein structure, dynamics, and thermodynamics. J Chem Theory Comput. 2010;6(3):890-909; doi: 10.1021/ct9004068.

7. Kaufmann KW, Lemmon G, DeLuca S, Sheehan JH, Meiler J. Practically useful: what the Rosetta protein modeling suite can do for you. Biochemistry. 2010;49(14): 2987-2998; doi: 10.1021/bi902153g.

8. Beauchamp KA, McGibbon R, Lin YS, Pande VS. Simple few-state models reveal hidden complexity in protein folding. Proc Natl Acad Sci U S A. 2012;109(44):1780717813; doi: 10.1073/pnas.1201810109.

9. Mishkind MC, Norr AM, Katz AC, Reger GM. Review of virtual reality treatment in psychiatry: evidence versus current diffusion and use. Curr Psychiatry Rep. 2017;19(11):80; doi: 10.1007/s11920-017-0836-0.

10. Klinger E, Légeron P, Roy S, Chemin I, Lauer F, Nugues P. Virtual reality exposure in the treatment of social phobia. Stud Health Technol Inform. 2004;99:91119; doi: 10.3233/978-1-60750-943-1-91.

11. Anderson PL, Price M, Edwards SM, Obasaju MA, Schmertz SK, Zimand E, et al. Virtual reality exposure therapy for social anxiety disorder: a randomized controlled trial. J Consult Clin Psychol. 2013;81(5): 751-760; doi: 10.1037/a0033559.

12. Li J, Theng YL, Foo S. Game-based digital interventions for depression therapy: a systematic review and metaanalysis. Cyberpsychol Behav Soc Netw. 2014;17(18): 519-527; doi: 10.1089/cyber.2013.0481.

13. Valmaggia LR, Latif L, Kempton MJ, Rus-Calafell M. Virtual reality in the psychological treatment for mental health problems: an systematic review of recent evidence. Psychiatry Res. 2016;236:189-195; doi: 10.1016/j. psychres.2016.01.015.

14. Kiper P, Szczudlik A, Venneri A, Stozek J, Luque-Moreno C, Opara J, et al. Computational models and motor learning paradigms: could they provide insights for neuroplasticity after stroke? An overview. J Neurol Sci. 2016;369:141-148; doi: 10.1016/j.jns.2016.08.019.

15. Deutsch JE, Merians AS, Adamovich S, Poizner H, Burdea GC. Development and application of virtual reality technology to improve hand use and gait of individuals post-stroke. Restor Neurol Neurosci. 2004;22(3-5): 371-386.

16. Subramanian SK, Lourenço CB, Chilingaryan G, Sveistrup H, Levin MF. Arm motor recovery using a virtual reality intervention in chronic stroke: randomized control trial. Neurorehabil Neural Repair. 2013;27(1):13-23; doi: 10.1177/1545968312449695.
17. Park J, Chung Y. The effects of robot-assisted gait training using virtual reality and auditory stimulation on balance and gait abilities in persons with stroke. NeuroRehabilitation. 2018;43(2):227-235; doi: 10.3233/ NRE-172415.

18. Jung SH, Song SH, Kim SD, Lee K, Lee GC. Does virtual reality training using the Xbox Kinect have a positive effect on physical functioning in children with spastic cerebral palsy? A case series. J Pediatr Rehabil Med. 2018;11(2):95-101; doi: 10.3233/PRM-160415.

19. Cameirão MS, Badia SB, Duarte E, Frisoli A, Verschure PF. The combined impact of virtual reality neurorehabilitation and its interfaces on upper extremity functional recovery in patients with chronic stroke. Stroke. 2012;43(10):2720-2728; doi: 10.1161/ STROKEAHA.112.653196.

20. Standen PJ, Threapleton K, Richardson A, Connell L, Brown DJ, Battersby S, et al. A low cost virtual reality system for home based rehabilitation of the arm following stroke: a randomised controlled feasibility trial. Clin Rehabil. 2017;31(3):340-350; doi: 10.1177/0269 215516640320.

21. Kiper P, Szczudlik A, Agostini M, Opara J, Nowobilski R, Ventura L, et al. Virtual reality for upper limb rehabilitation in subacute and chronic stroke: a randomized controlled trial. Arch Phys Med Rehabil. 2018; 99(5):834-842.e4; doi: 10.1016/j.apmr.2018.01.023.

22. Kiper P, Turolla A, Piron L, Agostini M, Baba A, Rossi S, et al. Virtual reality for stroke rehabilitation: assessment, training and the effect of virtual therapy. Med Rehabil. 2010;14(2):23-32.

23. Laver KE, George S, Thomas S, Deutsch JE, Crotty M. Virtual reality for stroke rehabilitation. Cochrane Database Syst Rev. 2015;2:CD008349; doi: 10.1002/1465 1858.CD008349.pub3.

24. Saposnik G, Cohen LG, Mamdani M, Pooyania S, Ploughman M, Cheung D, et al. Efficacy and safety of non-immersive virtual reality exercising in stroke rehabilitation (EVREST): a randomised, multicentre, singleblind, controlled trial. Lancet Neurol. 2016;15(10): 1019-1027; doi: 10.1016/S1474-4422(16)30121-1.

25. Borrego A, Latorre J, Llorens R, Alcañiz M, Noé E. Feasibility of a walking virtual reality system for rehabilitation: objective and subjective parameters. J Neuroeng Rehabil. 2016;13(1):68; doi: 10.1186/s12984016-0174-1.

26. Da Silva TF, de Franca ACL, de Souza MF, Silva AS. A single session of active video game play promotes postexercise hypotension in hypertensive middle-aged subjects. Hum Mov. 2018;19(2):82-89; doi: 10.5114/ hm.2018.74063.

27. De Vries AW, Faber G, Jonkers I, Van Dieen JH, Verschueren SMP. Virtual reality balance training for elderly: similar skiing games elicit different challenges in balance training. Gait Posture. 2018;59:111-116; doi: 10.1016/j.gaitpost.2017.10.006. 


\section{HUMAN MOVEMENT}

J. Mazurek et al., Virtual reality in healthcare

28. Dockx K, Alcock L, Bekkers E, Ginis P, Reelick M, Pelosin E, et al. Fall-prone older people's attitudes towards the use of virtual reality technology for fall prevention. Gerontology. 2017;63(6):590-598; doi: 10.1159/ 000479085.

29. Custură-Crăciun D, Cochior D, Constantinoiu S, Neagu C. Surgical virtual reality - highlights in developing a high performance surgical haptic device. Chirurgia. 2013;108(6):757-763.

30. Lin CS, Jeng MY, Yeh TM. The elderly perceived meanings and values of virtual reality leisure activities: a means-end chain approach. Int J Environ Res Public Health. 2018;15(4); doi: 10.3390/ijerph15040663. 